\title{
COMPLICAÇÕES DA TÉCNICA DE FIXAÇÃO TIBIAL COM PARAFUSO E ARRUELA PARA A RECONSTRUÇÃO LIGAMENTAR DO JOELHO
}

\author{
COMPLICATIONS OF THE SCREW/WASHER TIBIAL FIXATION TECHNIQUE \\ FOR KNEE LIGAMENT RECONSTRUCTION
}

\author{
Alexandre Almeida', Gilberto Roveda', Márcio Rangel Valin', Nayvaldo Couto de Almeida', \\ Vanderlei Sartor ${ }^{2}$, Soraya Melina Alves ${ }^{3}$
}

\section{RESUMO}

Objetivo: Avaliar a presença de dor ao nível da ferida operatória e a necessidade de retirada do parafuso de fixação tibial na reconstrução do ligamento cruzado anterior (LCA) do joelho e com o sexo e o índice de massa corporal (IMC). Métodos: Um grupo de 265 pacientes submetidos à reconstrução do LCA com enxerto ipsilateral dos tendões flexores da coxa em que o método de fixação tibial do enxerto foi o parafuso cortical com arruela metálica entre seis de julho de 2000 e 19 de novembro de 2007. Resultado: 176 pacientes foram avaliados com uma média de 33,3 \pm 19,5 meses (mediana de 29,5 meses) (IIQ: 17-45 meses) - mínima de oito e máxima de 87 meses. Não houve diferença estatística ao analisar a queixa de dor no parafuso $(p=0,272)$ e a necessidade de retirada do mesmo $(p=0,633)$ entre os sexos. Não houve diferença estatística ao analisar a queixa de dor no parafuso $(\mathrm{p}=0,08)$ e a necessidade de retirada do mesmo $(\mathrm{p}=$ 0,379 ) ao analisar o IMC. Conclusões: A utilização do parafuso e arruela metálica para fixação tibial na reconstrução do LCA demonstrou um índice de queixa de dor no sítio do parafuso na ordem de $25 \%$ e necessidade de sua retirada em $10,8 \%$ dos casos. Não houve predominância de queixas de dor na ferida operatória entre os sexos. Observou-se uma tendência a maior queixa de dor entre indivíduos com IMC $<25$. Não houve predominância de retirada do parafuso e da arruela entre os sexos ou entre indivíduos com diferentes IMC.

Descritores - Ligamento Cruzado Anterior; Artroscopia; Dor; Índice de massa corporal

\section{ABSTRACT}

Objective: To evaluate the presence of pain at the site of the surgical incision and the need to remove the tibial fixation screw after arthroscopic anterior cruciate ligament (ACL) reconstruction in relation to sex and body mass index (BMI). Methods: A group of 265 patients who underwent ACL reconstruction with ipsilateral flexor tendon grafts from the thigh using a screw and washer as the tibial fixation technique between July 2000 and November 2007. Results: 176 patients were evaluated for an average of $33.3 \pm 19.5$ months (median of 29.5 months) (IIQ: 17- 45 months) - minimum of 8 and maximum of 87 months. There was no statistical difference found upon analyzing complaints of pain at the site of the screw $(p=0.272)$ and the need to remove the tibial screw $(p=0.633)$ between sexes. No statistical differences were found concerning complaints of pain at the site of the screw $(p=0.08)$ and the need to remove the tibial screw $(p=0.379)$ according to BMI. Conclusion: The screw and metallic washer tibial method used for tibial fixation showed an incidence of pain complaints of $25 \%$, and the need for its removal in $10.8 \%$ of the cases. There was no prevalence of complaints of pain at the surgical wound between the sexes. There was a greater tendency to complain about pain among patients with $B M I<25$. There was no predominance of removal of the screw and washer between the sexes and individuals with different BMIs.

Keywords - Anterior Cruciate Ligament; Arthroscopy; Body mass index; Pain

\footnotetext{
1 - Médico Ortopedista do Hospital Saúde de Caxias do Sul, RS.

2 - Médico Residente do Serviço de Ortopedia e Traumatologia do Hospital Pompeia de Caxias do Sul, RS.

3 - Acadêmica do $6^{\circ}$ Ano do Curso de Medicina da Universidade de Caxias do Sul, RS.
}

Trabalho realizado no Hospital Saúde de Caxias do Sul, RS.

Correspondência: Rua Vitório Buzelatto, 222/601, Bairro Madureira - 95020-290 - Caxias do Sul, RS - E-mail: bone@visao.com.br 


\section{INTRODUÇÃO}

A reconstrução do ligamento cruzado anterior (LCA) com o autoenxerto dos tendões flexores da coxa está demonstrada como uma técnica segura e com bons resultados ${ }^{(1-4)}$. Há discussão na literatura quanto ao método ideal para a realização do procedimento no que diz respeito à escolha da técnica cirúrgica (intra-articular isolada, extra-articular e intra-articular com reforço extra-articular), escolha do enxerto, escolha do método de fixação e resultado final ${ }^{(1,2,5,6)}$.

O enxerto duplo dos tendões flexores da coxa e o tendão patelar dividem as preferências para escolha. Vários estudos têm demonstrado resultados semelhantes quanto à estabilidade comparando os dois enxertos, havendo uma tendência para o uso dos tendões flexores, pois pacientes submetidos a esta técnica apresentam menor queixa de dor anterior no joelho pós-operatória ${ }^{(2-4,7-9)}$. Nas metaanálises publicadas após o ano 2000, foi demonstrado que as técnicas que envolvem o tendão patelar parecem ter melhor capacidade para alcançar um joelho estável quando comparadas às técnicas de reconstrução com os tendões flexores da coxa; no entanto, à custa de um maior número de complicações e, novamente, de dor anterior no joelho ${ }^{(3,4)}$.

Os métodos de fixação variam muito. Kurosaka et $a l^{(10)}$ observaram que o sítio de fixação é o elo mecanicamente mais fraco do ligamento reconstruído. A fixação tibial é a literatura apresenta maior controvérsia em definir o melhor implante a ser utilizado. Magen et $a l^{(11)}$ estudaram seis tipos de fixação de enxertos tendinosos livres na tíbia e concluíram que a amarrilha em um parafuso e arruela é o melhor método quando foram avaliadas a rigidez e a resistência à tração.

A região anatômica anteromedial na tíbia é escassa de tecido celular subcutâneo. A hipótese que estimulou este estudo foi a de que pacientes magros e do sexo feminino teriam maiores queixas ao nível da fixação tibial e consequentemente necessitariam de cirurgia para retirada da síntese. O objetivo desta pesquisa é avaliar a presença de dor ao nível da ferida operatória, bem como a necessidade de retirada do material de síntese em pacientes submetidos à fixação tibial com parafuso e arruela e relacioná-las com o sexo e o índice de massa corporal (IMC).

\section{MÉTODOS}

Foi analisado, de forma transversal, um grupo de 265 pacientes submetidos à artroscopia do joelho para fins de reconstrução do LCA com enxerto ipsilateral dos tendões flexores da coxa no período de seis de julho de 2000 a 19 de novembro de 2007.

O paciente eleito para a reconstrução ligamentar apresentava queixa de instabilidade do joelho, dificuldades para prática desportiva ou dificuldades para o desempenho profissional.

O procedimento foi executado sempre pelo mesmo cirurgião.

Técnica: Após realizado o bloqueio subaracnoideo com bupivacaína pesada e adição de morfina, o paciente era posicionado em decúbito dorsal e o membro inferior colocado sob garroteamento pneumático. Eram retirados e pré-tensionados em mesa especial os autoenxertos ipsilaterais do gracilis e semitendíneo, procedimento realizado por uma incisão de $3 \mathrm{a} 4 \mathrm{~cm}$ ao nível de sua inserção tibial. Os túneis ósseos necessários para a reconstrução do LCA eram confeccionados sob visão artroscópica. A fixação femoral foi realizada com endobutton e a tibial através da amarrilha com fios inabsorvíveis de Ethibond ${ }^{\circledR} 5.0$ previamente fixados às quatro extremidades livres dos enxertos em um parafuso metálico cortical de $4,5 \mathrm{~mm}$ com uma arruela metálica tipo AO lisa com diâmetro de $8 \mathrm{~mm}$ e espessura de $2 \mathrm{~mm}$ (Figura 1).

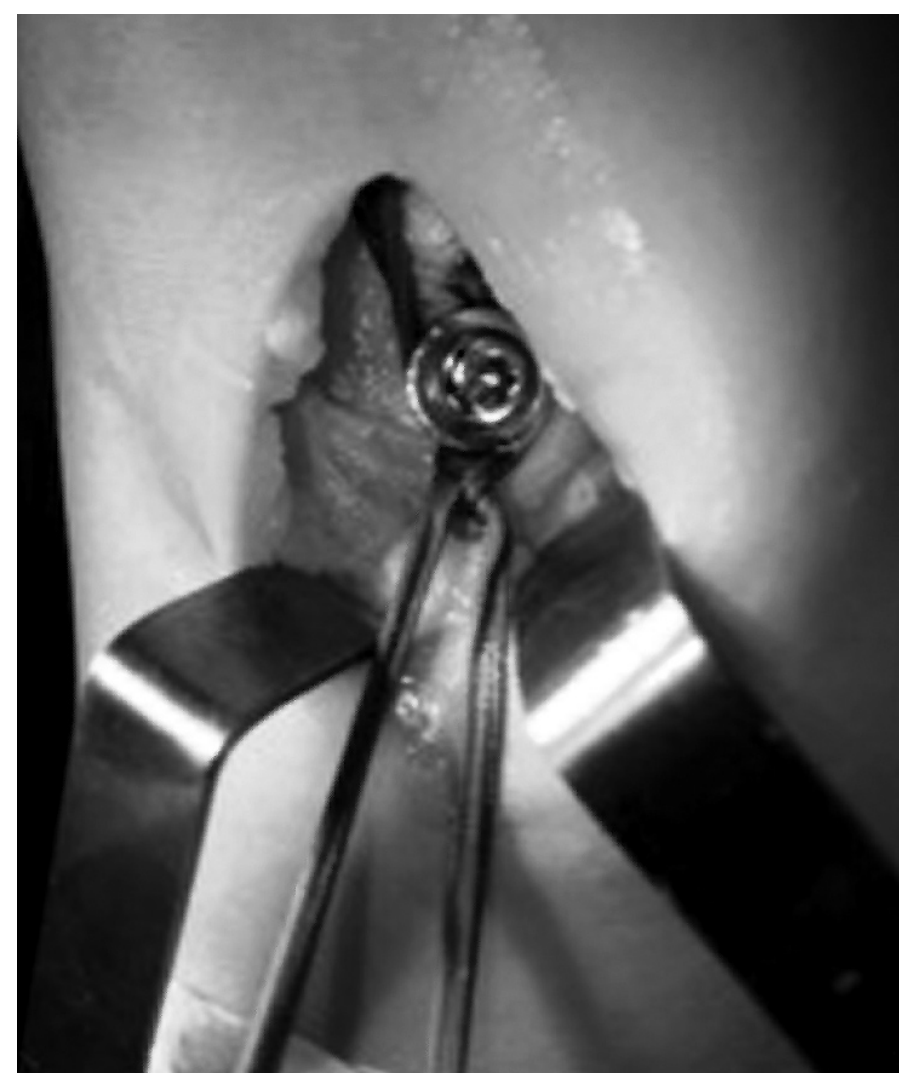

Figura 1 - Fixação tibial em poste. 
A ferida operatória foi fechada por planos. Não foi utilizado dreno de aspiração. Um enfaixamento compressivo era aplicado após a realização do procedimento cirúrgico, sendo que o paciente, os familiares e a enfermagem eram orientados a manter o membro inferior em extensão completa ${ }^{(12)}$ (Figuras 2 e 3). A crioterapia, como método adjuvante, foi utilizada em todos os $\operatorname{casos}^{(13)}$.

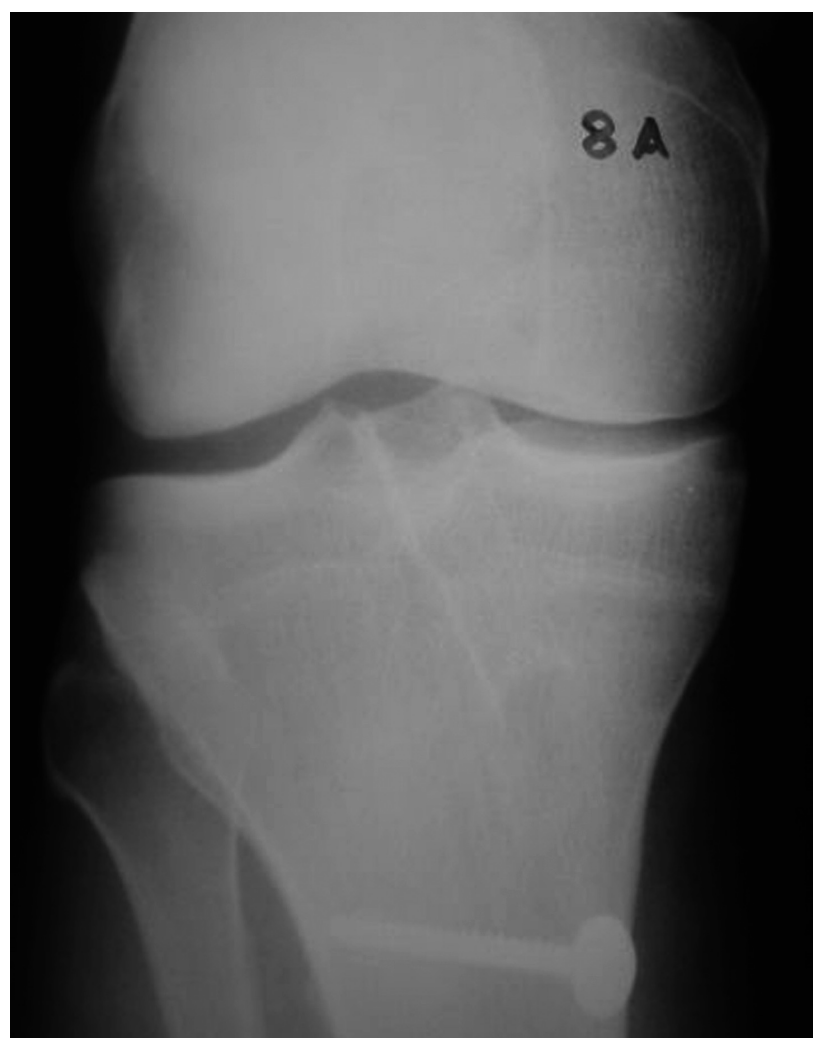

Figura 2 - Radiografia pós-operatória em AP.

Os exercícios de elevação ativa do MI eram iniciados tão logo o paciente recuperava o controle dos MMII. O apoio era protegido por três semanas, quando iniciavam os exercícios de cadeia cinética fechada. Os exercícios de cadeia cinética aberta eram permitidos aos quatro meses de PO. O retorno ao gesto esportivo iniciava aos seis meses, com recuperação total das atividades aos oito meses de pós-operatório.

Foram excluídos deste estudo todos os pacientes que apresentavam ao nível da metáfise proximal da tíbia a presença de outro material de síntese (haste tibial com parafuso de bloqueio proximal, placa de Puddu e outros) que pudesse comprometer a avaliação. Também foram excluídos quaisquer pacientes com alguma condição preexistente que pudesse comprometer o resultado (perda muscular por sequela de queimadura) e os pacientes com menos de oito meses de pós-operatório.

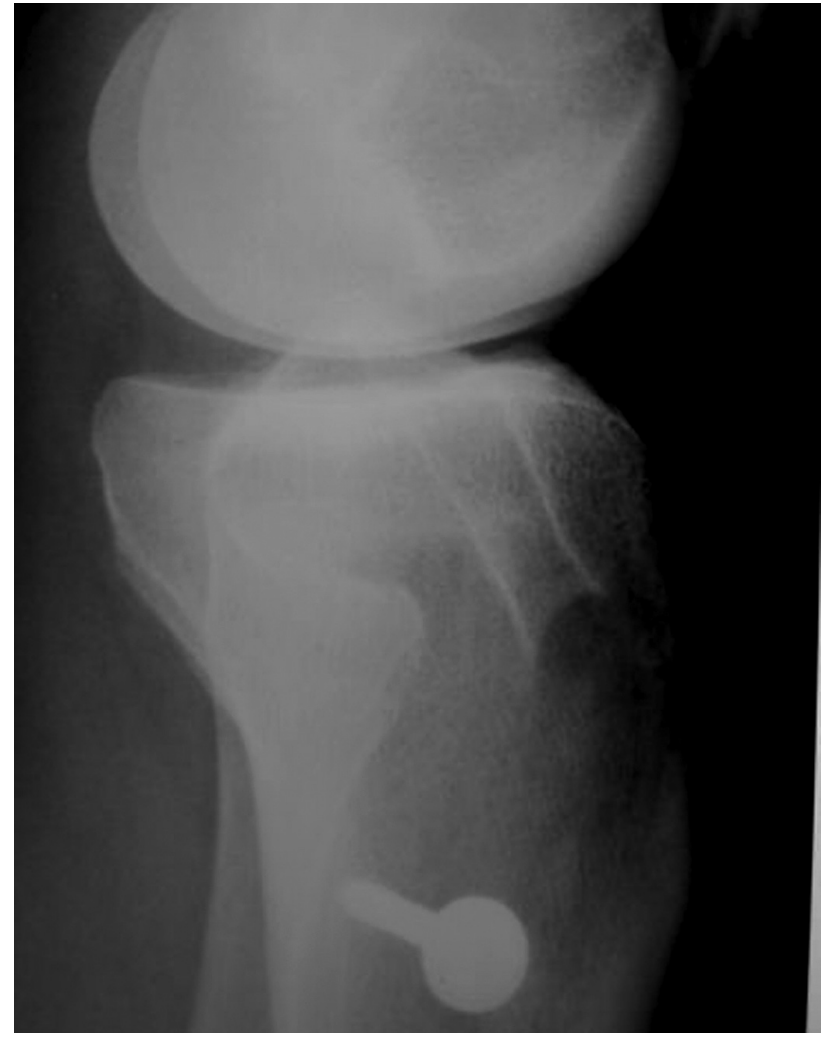

Figura 3 - Radiografia pós-operatória em perfil.

Os pacientes foram submetidos à medição da altura e do peso no pré-operatório imediato, para cálculo do IMC como determina a rotina da instituição onde o procedimento foi realizado. As medidas foram realizadas através do mesmo instrumental, com o paciente vestindo apenas a camisola do centro cirúrgico.

O IMC $\left(\mathrm{kg} / \mathrm{m}^{2}\right)$ foi utilizado para classificar os pacientes de acordo com o grau de obesidade ${ }^{(14)}$, conforme a Tabela 1 .

As variáveis estudadas foram: sexo, IMC, dor ao nível da ferida operatória, necessidade de procedimento adicional para a retirada do parafuso de fixação.

Os dados foram analisados por meio do conjunto estatístico SPSS (Statistical Package for Social Sciences) versão 12.0 (SPSS Inc. 1989-2003). Para a análise estatística, foram utilizados: cálculo das médias, desvio

Tabela 1 - Graus de obesidade.

\begin{tabular}{c|c}
\hline \multicolumn{2}{c}{ Classificação da obesidade } \\
\hline \multicolumn{2}{c}{ IMC $(\mathrm{kg} / \mathrm{m} 2)$} \\
\hline $18-24,9$ & Normal \\
\hline $25-29,9$ & Sobrepeso \\
\hline $30-34,9$ & Obesidade $\mathrm{G} 1$ \\
\hline $35-39,9$ & Obesidade G2 \\
\hline$>=40,0$ & Obesidade mórbida \\
\hline
\end{tabular}

Legenda: IMC - índice de massa corporal; $\mathrm{kg} / \mathrm{m} 2$ - unidade de medida do índice de massa corporal. 
padrão, mediana, frequência e percentual. Utilizou-se o teste $t$ de Student e o ANOVA de uma via para comparar as médias das variáveis simétricas. Os testes não paramétricos de Mann-Whitney e Kruskal-Wallis foram utilizados para análise das variáveis assimétricas e o teste do $X^{2}$ (Qui-quadrado) para a comparação de frequências. Foram consideradas significantes as diferenças com $\mathrm{p} \leq$ 0,05 para um intervalo de confiança de $95 \%$.

\section{RESULTADOS}

O estudo avaliou 265 pacientes, dos quais foram excluídos 78 de acordo com os critérios de exclusão, totalizando um $\mathrm{n}=187$ pacientes. Houve uma perda amostral de $11(5,88 \%)$ pacientes, sendo dois por óbito e nove por falha no acompanhamento.

Um total de 176 pacientes foram avaliados com uma média de 33,3 \pm 19,5 meses (mediana de 29,5 meses) (IIQ: 17-45 meses). A avaliação mínima foi aos oito meses e a máxima aos 87 meses.

A média de idade foi de $32,2 \pm 9,8$ anos.

Nesta amostra, 131 pacientes eram masculinos $(74,4 \%)$ e 45 femininos (25,6\%). A média de idade para o sexo masculino foi de $32,2 \pm 9,4$ anos e para o feminino foi de $32,3 \pm 11,2$ anos. Os números de joelhos direitos operados foi $80(45,5 \%)$ e de esquerdos foi $96(54,5 \%)$.

Houve queixa de dor ao nível da ferida operatória, na qual ficava localizado o parafuso de fixação tibial com a arruela metálica, em 44 (25\%) pacientes. O número de pacientes do sexo masculino com queixa na ferida operatória foi de 30/131 (22,9\%) e femininos foi de $14 / 45(31,1 \%)$.

Ao analisar a queixa de dor no parafuso de fixação tibial com a arruela metálica entre os sexos não verificamos diferença estatística significativa $(p=0,272)$.

Houve necessidade de retirada do parafuso de fixação tibial e da arruela em 19 (10,8\%) pacientes, sendo estes $15(78,9 \%)$ masculinos e quatro femininos $(21,1 \%)$. O momento de retirada do material de síntese variou entre quatro e 54 meses de pós-operatório, com uma média de 14,5 $\pm 10,8$ meses (mediana de 11 meses) (IIQ: 8-18 meses).

Ao analisar a necessidade de retirada do parafuso de fixação tibial e da arruela entre os sexos verificamos não haver diferença estatística significativa $(\mathrm{p}=0,633)$.

Com relação ao grau de obesidade, $76(43,2 \%)$ pacientes apresentavam o peso considerado como normal, 69 (39,2\%) pacientes apresentavam sobrepeso, 27 $(15,3 \%)$ pacientes foram classificados como obesidade grau um e quatro $(2,3 \%)$ pacientes foram classificados como obesidade grau dois. Não houve a presença de pacientes com obesidade mórbida nesta amostra.

Foi verificada a presença de dor ao nível da ferida operatória em 24/76 (31,57\%) pacientes com IMC considerado normal (IMC < 25). Ao agrupar os pacientes com IMC > 25 (sobrepeso, obesidade grau um e obesidade grau dois) foi verificada a presença de dor na ferida operatória em 20/100 (20\%) pacientes. Não houve diferença estatística significativa com relação à presença de dor ao nível da ferida operatória quando comparados os dois grupos $(p=0,08)$.

Foi verificada a necessidade de retirar o parafuso de fixação tibial e da arruela em 10/76 (13,2\%) pacientes com IMC considerado normal (IMC < 25). Ao agrupar os pacientes com IMC $>25$ (sobrepeso, obesidade grau um e obesidade grau dois) foi verificada a necessidade de retirar o parafuso de fixação tibial e da arruela em 9/100 (9\%) pacientes. Não houve diferença estatística significativa com relação à necessidade de retirar o parafuso de fixação tibial e da arruela quando comparados os dois grupos $(\mathrm{p}=0,379)$.

\section{DISCUSSÃO}

A literatura é ampla no que diz respeito aos estudos que têm por objetivo pesquisar qual seria o substituto ideal para a complexa estrutura que representa o $\operatorname{LCA}^{(1,2,5-7,15-19)}$.

O uso do tendão patelar (TP) para a reconstrução do LCA por muito tempo foi considerado o padrão ouro, porém a técnica não é isenta de complicações. Entre as complicações atribuídas, encontram-se: a dor pósoperatória na área doadora do enxerto ${ }^{(7)}$, a dor anterior no joelho $^{(20,21)}$, a dor para ajoelhar ${ }^{(20)}$, anestesia cutânea na ferida operatória ${ }^{(21)}$, a diminuição de força do mecanismo extensor do joelho ${ }^{(20,21)}$, a ruptura do tendão patelar ${ }^{(20)}$, a fratura da patela ${ }^{(20)}$ e a tendinite patelar ${ }^{(20,21)}$.

A dor na área doadora do TP (face anterior do joe1ho) está relatada na literatura em até $40 \%{ }^{(20,21)}$. Deehan et $a l^{(22)}$ encontraram $8 \%$ crepitação sintomática na articulação femoropatelar de sua casuística.

Uma variação da técnica que busca o enxerto no mecanismo extensor do joelho é a retirada do tendão quadricipital (TQ). Fulkerson e Langeland ${ }^{(23)}$ reportam $45 \%$ de incidência de dor anterior no joelho c/ o TQ. Cortelazo et al ${ }^{(24)}$ encontraram desconforto ao ajoelhar em $44 \%$ de seus pacientes após cinco anos da cirurgia de reconstrução do LCA com o enxerto do TQ. 
Na pesquisa por um enxerto menos agressivo surgiu a tendência para a reconstrução do LCA com os tendões flexores da coxa. Esta técnica reduziu de forma significativa as queixas de dor anteriormente citadas para o uso do TP e TQ através da diminuição da morbidade do procedimento $^{(25-27)}$.

Utilizamos a técnica de reconstrução do LCA com o enxerto autólogo dos tendões grácil e semitendíneo desde o ano 2000. O principal motivo para a migração definitiva a partir da técnica do TP foi a verificação da menor dor pós-operatória ${ }^{(25)}$.

A fixação distal do enxerto dos tendões flexores ao nível da metáfise tibial é considerada o elo fraco da reconstrução do LCA e se tornou um desafio para os estudiosos de biomecânica devido à menor densidade óssea deste local ${ }^{(28,29)}$. Atualmente encontram-se disponíveis no mercado vários tipos de implantes divididos em fixação intratúnel e fixação cortical. Este amplo arsenal terapêutico tem a possibilidade de modificar de forma significativa o custo do procedimento cirúrgico.

A fixação intratúnel é utilizada pela praticidade de uso e pelo baixo índice de queixas locais ${ }^{(30)}$. Sua resistência à tração é dependente da qualidade do osso metafisário e alguns estudos sugerem o uso de alguma fixação adicional para atingir o sucesso da reconstrução ligamentar $^{(31)}$. Hill et $a l^{(31)}$ propuseram a utilização de um grampo cortical, enquanto outros autores sugerem a utilização de enxerto ósseo intratúnel ${ }^{(32,33)}$ e ainda o cuidado com a técnica no momento da inserção do parafuso para não perder a tensão no enxerto, como foi levantado por Grover et $a l^{(34)}$.

A fixação cortical dos tendões flexores é reconhecida por fornecer grande rigidez ao sistema com o inconveniente de gerar queixas de irritação no subcutâneo em alguns $\operatorname{casos}^{(29)}$. Hill et $a l^{(31)}$, utilizando o grampo cortical para aumentar a rigidez do sistema de fixação, verificaram a presença de dor ao ajoelhar em $29 \%$ dos seus pacientes.

Um outro método de fixação cortical é a utilização da amarrilha de fios não absorvíveis em um parafuso cortical com arruela metálica. Este método de fixação, também chamado de "fixação em poste", foi considerado a melhor forma de fixação do LCA entre seis diferentes métodos testados por Magen et $a^{(11)}$ no que diz respeito à rigidez da fixação. Embora seja um método seguro, barato e adequado para fixação dos enxertos livres dos tendões flexores, aumenta o relevo da cortical anteromedial da tíbia, fazendo com que a saliência da cabeça do parafuso possa ser percebida pelo paciente com certa facilidade. Tal fato é particularmente importante quando o paciente é magro e do sexo feminino, gerando queixas álgicas e estéticas e havendo necessidade de retirada do parafuso em alguns $\operatorname{casos}^{(11,35,36)}$. Nossa casuística não demonstrou de forma significativa que as mulheres tenham maior risco de apresentar queixa local $(p=0,272)$ ou maior necessidade de retirada da síntese $(\mathrm{p}=0,633)$.

Asano et $a l^{(37)}$, em estudo investigando a evolução das lesões condrais no pós-operatório da reconstrução do LCA, aproveitaram o procedimento de retirada do parafuso e da arruela na cortical anterior da tíbia para fazerem um secound look nos seu pacientes. Informaram que a queixa de dor e a vontade de retirar o material de síntese foi o fator motivador do segundo procedimento. Seu índice de retirada do material de síntese foi de $66,3 \%$.

Em nosso estudo, encontramos a queixa de dor e desconforto no joelho relacionada ao material de síntese em $25 \%$ dos pacientes. Tivemos o cuidado de analisar o perfil destes pacientes e observamos uma tendência à queixa de dor local nos pacientes magros $(\mathrm{p}=0,08)$. No entanto, a evolução para um procedimento cirúrgico adicional no qual o parafuso seria retirado ocorreu em apenas $10,8 \%$ dos casos $(p=0,379)$.

É unanimidade na literatura que a escolha do tipo de enxerto e da técnica de fixação deve ser individualizada à condição de cada paciente e, principalmente, à preferência do cirurgião, o qual deve considerar seu conhecimento e sua experiência sobre cada técnica.

As variações do peso corporal encontradas em alguns pacientes nos pós-operatórios estudados podem ser consideradas um viés do estudo, uma vez que pacientes podem mudar de grupo de IMC nos meses e anos que seguiram o procedimento cirúrgico.

\section{CONCLUSÕES}

A reconstrução do LCA com enxerto dos tendões flexores da coxa e fixação tibial com parafuso e arruela demonstrou um índice de queixa de dor no sítio do parafuso na ordem de $25 \%$. Houve necessidade de retirada do parafuso de fixação tibial e da arruela em $10,8 \%$ dos casos.

Não houve predominância de queixas de dor na ferida operatória entre os sexos. Observou-se uma tendência maior à queixa de dor entre indivíduos com IMC menor do que 25 .

Não houve predominância de retirada do parafuso e da arruela entre os sexos ou entre indivíduos com diferentes IMC. 


\section{REFERÊNCIAS}

1. Prodromos CC, Han YS, Keller BL, Bolyard RJ. Stability results of hamstring anterior cruciate ligament reconstruction at 2- to 8-year follow-up. Arthroscopy. 2005;21(2):138-46.

2. Aglietti P, Giron F, Buzzi R, Biddau F, Sasso F. Anterior cruciate ligament reconstruction: bone-patellar tendon-bone compared with double semitendinosus and gracilis tendon grafts. A prospective, randomized clinical trial. J Bone Joint Surg Am. 2004;86(10):2143-55

3. Freedman KB, D'Amato MJ, Nedeff DD, Kaz A, Bach BR Jr. Arthroscopic anterior cruciate ligament reconstruction: a metaanalysis comparing patellar tendon and hamstring tendon autografts. Am J Sports Med. 2003;31(1):2-11.

4. Yunes M, Richmond JC, Engels EA, Pinczewski LA. Patellar versus hamstring tendons in anterior cruciate ligament reconstruction: A meta-analysis. Arthroscopy. 2001;17(3):248-57.

5. Barrett GR, Papendick L, Miller C. Endobutton button endoscopic fixation technique in anterior cruciate ligament reconstruction. Arthroscopy. 1995;11(3):340-3

6. Hoffmann F, Friebel $\mathrm{H}$, Schiller M. [The semitendinosus tendon as replacement for the anterior cruciate ligament]. Zentralbl Chir. 1998;123(9):994-1001.

7. Laxdal G, Kartus J, Hansson L, Heidvall M, Ejerhed L, Karlsson J. Aprospective randomized comparison of bone-patellar tendon-bone and hamstring grafts for anterior cruciate ligament reconstruction. Arthroscopy. 2005;21(1):34-42.

8. Aglietti P, Buzzi R, Zaccherotti G, De Biase P. Patellar tendon versus doubled semitendinosus and gracilis tendons for anterior cruciate ligament reconstruction. Am J Sports Med. 1994 Mar-Apr;22(2):211-7.

9. Callway G, Nicholas S, Lavannaugh J. Hamstring augmentation versus patella tendon reconstruction of acute ACL disruption: a randomized prospective study. In: 61st Annual Meeting of the American Academy of Orthopaedic Surgeons, New Orleans;1994

10. Kurosaka M, Yoshiya S, Andrish JT. A biomechanical comparison of different surgical techniques of graft fixation in anterior cruciate ligament reconstruction. Am J Sports Med. 1987;15(3):225-9.

11. Magen HE, Howell SM, Hull ML. Structural properties of six tibial fixation methods for anterior cruciate ligament soft tissue grafts. Am J Sports Med. 1999;27(1):35-43.

12. Pförringer W, Kremer C. [Subsequent treatment of surgically managed, fresh anterior cruciate ligament ruptures--a randomized, prospective study]. Sportverletz Sportschaden. 2005;19(3):134-9.

13. Raynor MC, Pietrobon R, Guller U, Higgins LD. Cryotherapy after ACL reconstruction: a meta-analysis. J Knee Surg. 2005;18(2):123-9.

14. Mancini MC. Noções Fundamentais - Diagnóstico e Classificação da Obesidade. In: Garrido Junior AB, Ferraz EM, Barroso FL, Marchesini JB, Szego T. Cirurgia da obesidade. São Paulo:Atheneu; 2002. p. 1-7.

15. Ma CB, Francis K, Towers J, Irrgang J, Fu FH, Harner $\mathrm{CH}$. Hamstring anterior cruciate ligament reconstruction: a comparison of bioabsorbable interference screw and endobutton-post fixation. Arthroscopy. 2004;20(2):122-8.

16. O'Neill DB. Arthroscopically assisted reconstruction of the anterior cruciate ligament. A follow-up report. J Bone Joint Surg Am. 2001;83(9):1329-32.

17. Corry IS, Webb JM, Clingeleffer AJ, Pinczewski LA. Arthroscopic reconstruction of the anterior cruciate ligament. A comparison of patellar tendon autograft and four-strand hamstring tendon autograft. Am J Sports Med. 1999;27(4):444-54.

18. Rodeo SA, Arnoczky SP, Torzilli PA, Hidaka C, Warren RF. Tendon-healing in a bone tunnel. A biomechanical and histological study in the dog. J Bone Joint Surg Am. 1993;75(12):1795-803.

19. Miller SL, Gladstone JN. Graft selection in anterior cruciate ligament reconstruction. Orthop Clin North Am. 2002;33(4):675-83.
20. Sachs RA, Daniel DM, Stone ML, Garfein RF. Patellofemoral problems after anterior cruciate ligament reconstruction. Am J Sports Med. 1989;17(6):760-5.

21. Shino K, Nakagawa S, Inoue M, Horibe S, Yoneda M. Deterioration of patellofemoral articular surfaces after anterior cruciate ligament reconstruction. Am J Sports Med. 1993;21(2):206-11.

22. Deehan DJ, Salmon LJ, Webb VJ, Davies A, Pinczewski LA. Endoscopic reconstruction of the anterior cruciate ligament with an ipsilateral patellar tendon autograft. A prospective longitudinal five-year study. J Bone Joint Surg Br. 2000;82(7):984-91.

23. Fulkerson JP, Langeland $\mathrm{RH}$. The central quadriceps tendon graft for cruciate ligament reconstruction. Oper Thech Orthop. 1996;6:135-7.

24. Cortelazo MJ, Cohen M, Mestriner LA, Carneiro Filho M. Reconstrução artroscópica do ligamento cruzado anterior com enxerto do tendão quadricipital: estudo das características dimensionais do tendão e da técnica cirúrgica. Rev Bras Ortop. 2002;37(6):247-55.

25. Almeida A, Valin MR, Almeida NC, Ferreira R. Avaliação da dor após a reconstrução artroscópica do ligamento cruzado anterior do joelho. Rev Bras Ortop. 2006;41(8):320-24.

26. Clark R, Olsen RE, Larson BJ, Goble EM, Farrer RP. Cross-pin femoral fixation: a new technique for hamstring anterior cruciate ligament reconstruction of the knee. Arthroscopy. 1998;14(3):258-67.

27. Colombet $\mathrm{P}$, Allard M, Bousquet V, de Lavigne C, Flurin PH, Lachaud C. Anterior cruciate ligament reconstruction using four-strand semitendinosus and gracilis tendon grafts and metal interference screw fixation. Arthroscopy. 2002;18(3):232-7.

28. Paulos LE, Stewart AM. Tibial fixation for hamstring anterior cruciate ligament reconstruction. Tech Orthop. 2005;20(3):303-5

29. Prodromos C. Low-profile cortical screw tibial fixation for hamstring anterior cruciate ligament reconstruction: surgical technique and stability results. Techn Orthop. 2005;20(3):272-3.

30. Bartz RL, Mossoni K, Tyber J, Tokish J, Gall K, Siparsky PN. A biomechanical comparison of initial fixation strength of 3 different methods of anterior cruciate ligament soft tissue graft tibial fixation: resistance to monotonic and cyclic loading. Am J Sports Med. 2007;35(6):949-54

31. Hill PF, Russell VJ, Salmon LJ, Pinczewski LA. The influence of supplementary tibial fixation on laxity measurements after anterior cruciate ligament reconstruction with hamstring tendons in female patients. Am J Sports Med. 2005;33(1):94-101.

32. Matsumoto A, Howell SM. The WasherLoc and Bone Dowel: a rigid, slippageresistant tibial fixation device for a soft tissue anterior cruciate ligament graft. Tech Orthop. 2005;20(3):278-82.

33. Howell SM, Roos P, Hull ML. Compaction of a bone dowel in the tibial tunnel improves the fixation stiffness of a soft tissue anterior cruciate ligament graft: an in vitro study in calf tibia. Am J Sports Med. 2005;33(5):719-25.

34. Grover DM, Howell SM, Hull ML. Early tension loss in an anterior cruciate ligament graft. A cadaver study of four tibial fixation devices. J Bone Joint Surg Am. 2005;87(2):381-90.

35. Martin SD, Martin TL, Brown CH. Anterior cruciate ligament graft fixation. Orthop Clin North Am. 2002;33(4):685-96.

36. Allen CR, Giffin JR, Harner CD. Revision anterior cruciate ligament reconstruction. Orthop Clin North Am. 2003;34(1):79-98.

37. Asano H, Muneta T, Ikeda H, Yagishita K, Kurihara Y, Sekiya I. Arthroscopic evaluation of the articular cartilage after anterior cruciate ligament reconstruction: a short-term prospective study of 105 patients. Arthroscopy. 2004;20(5):474-81. 